Bayero Journal of Pure and Applied Sciences, 5(2): 156 - 159

Received: June 2012

Accepted: August 2012

ISSN $2006-6996$

\title{
KYNURENINE AND SEROTONIN PATHWAYS: A REVIEW
}

\author{
Adegbusi, H.S.
}

Department of Computer Science, Katsina University, PMB 2137 Katsina

Correspondence author: Khalidadegbusi@yahoo.com

\begin{abstract}
This paper seeks to elucidate the two-key pathways involving tryptophan matabolism, namely, kynurenine and serotonin pathways. In the kynurenine pathway (KP), the discussion considers the steps of the oxidative degradation of tryptophan to yield nicotinate mononucleotide, a precursors for the biosynthesis of nicotinate nucleotides ( NAD ${ }^{+}$and NADP ${ }^{+}$), while serotonin pathway (SP) considers the biosynthesis of serotonin from tryptophan. Two different isoforms of tryptophan hydroxylase (TPH) are involved in SP: Tryptophan hydroxylase -1 (TPH ${ }_{1}$ catalysis SP in enterochromaffin cells of the gut, while Tryptophan hydroxylase-2 (TPH $)$ catalysis SP in the nerve cells of the central nervous system, the brain. Emphasis is given to the relevance of pyridoxal phosphate (PLP) in KP and also identified in the pathway of KP is the liberation of alanine as a biproduct, a basis for the gluconeogenecity of tryptophan. Serotonin, a major bioactive end-product of SP is a potent neurotransmitter, vasoconstrictor, regulation of intestinal motility and a player in cognitive function. Foods that give an increased ratio of tryptophan to phenylalanine and leucine such as nuts of walnut, plantains, bananas, dates, pineapples and tomatoes are good sources of serotonin. Research suggests a diet rich in carbohydrates and low protein is also a good source. Owning to the significance of some the major bioactive end-products, intermediates and byproducts of these pathways for the well-being and happiness of human beings, more research should be carried out therein not only to unravel some of the disorders they may be associated with but to also help in the development and production of; therapeutic drugs for psychiatric and sexual disorders, and intestinal antidote.
\end{abstract}

Keywords: Nicotinate mononucleotide, serotonin, kynurenine, dietary source of serotonin.

\section{INTRODUCTION}

It is only in recent years that kynurenine pathway (KP) emerged from relative obscurity to feature prominently in research associated with psychiatric disorder and regulation of the immune system (Pattern, 2012). KP is a metabolic pathway leading to the production of nicotinamide adenine dinucleotide $\left(\mathrm{NAD}^{+}\right)$(Wikipedia, 2012a) and nicotinamide adenine dinucleotide phosphate $\left(\mathrm{NADP}^{+}\right.$) (Satyanarayana and Chakrapani, 2010) from the degradation of tryptophan.

Kynurenine pathway mostly occurs in the liver (satyanarayana and Chakrapani, 2010) and involves physiological functions such as behavior, sleep, thermoregulation and pregnancy (Wikipedia, 2012a). There are also evidences of the pathway involvement in neurotoxic mechanisms associated with several inflammatory neurological diseases including AIDS-dementias complex, Alzheimer's, Huntington's and Parkinson's diseases, amyotrophic lateral and multiple sclerosis, depression and schizophrenia (Wikipedia, 2012a). The intermediates of KP have diverse biological effects, with certain intermediates acting as neurotoxins, others as neuroprotective agents and still others as reactive oxidative species (Pattern, 2012). Therefore, the relative balance of intermediate is important when interpreting patterns of the KP.

$\mathrm{KP}$ is affected by such factors as B-vitamin deficiencies (stone, 2001), immune activation, polyunsaturated fatty acid intake, phthalate exposure, protein intake and corticosteroids (Pattern, 2012). KP and serotonin pathways (SP) are two different closely related pathways because tryptophan is their initial substrate. $\mathrm{SP}$ is involved in the biosynthesis of serotonin from tryptophan (Walther et al., 2003). Serotonin or 5hydroxytryptamine $(5 \mathrm{HT})$ is a neurotransmitter primarily found in the gastrointestinal tract, platelets, and in the central nervous system (CNS) of animals including humans (Young, 2007). About 1\% of tryptophan is converted to serotonin (Satyanarayana and Chakrapani, 2006) but in individuals with imbalances in immune system, tryptophan may not be available for serotonin synthesis and thus contribute to impaired quality of life and depressive mood (Pattern, 2012). Extremely high levels of serotonin, though are essentially impossible to reach, can cause a condition known as serotonin syndrome, with toxic and potentially fatal effect; low levels of serotonin resulted in low heart and breathing rates in experimental rats and hence a root cause of sudden infant death syndromes (SIDs); and in depressed patients and animals that model the disorder, levels of serotonin are decreased (Wikipedia, 2012b). Furthermore, modulation of serotonin at synapses is thought to be a major action of several classes of pharmacological antidepressants (Wikipedia, 2012b). It is mainly metabolized to 5 -Hydroxylindole acetate (5HIAA), chiefly by the liver. 
Owing to the novelty of KP in the academic research and the roles of serotonin in human well-being, this paper is thus prepared to underscore these pathways so that more research can be carried out in their respect not only to unravel some of the genetic and or metabolic disorders they may be associated with but to also harness some of the effects of serotonin in animals, especially man, for drugs development and production.

\section{KYNURENINE AND SEROTONIN PATHWAYS}

Kynurerine Pathway:

Figure 1 shows the various intermediates and enzymes which make up Kynurenine Pathway:
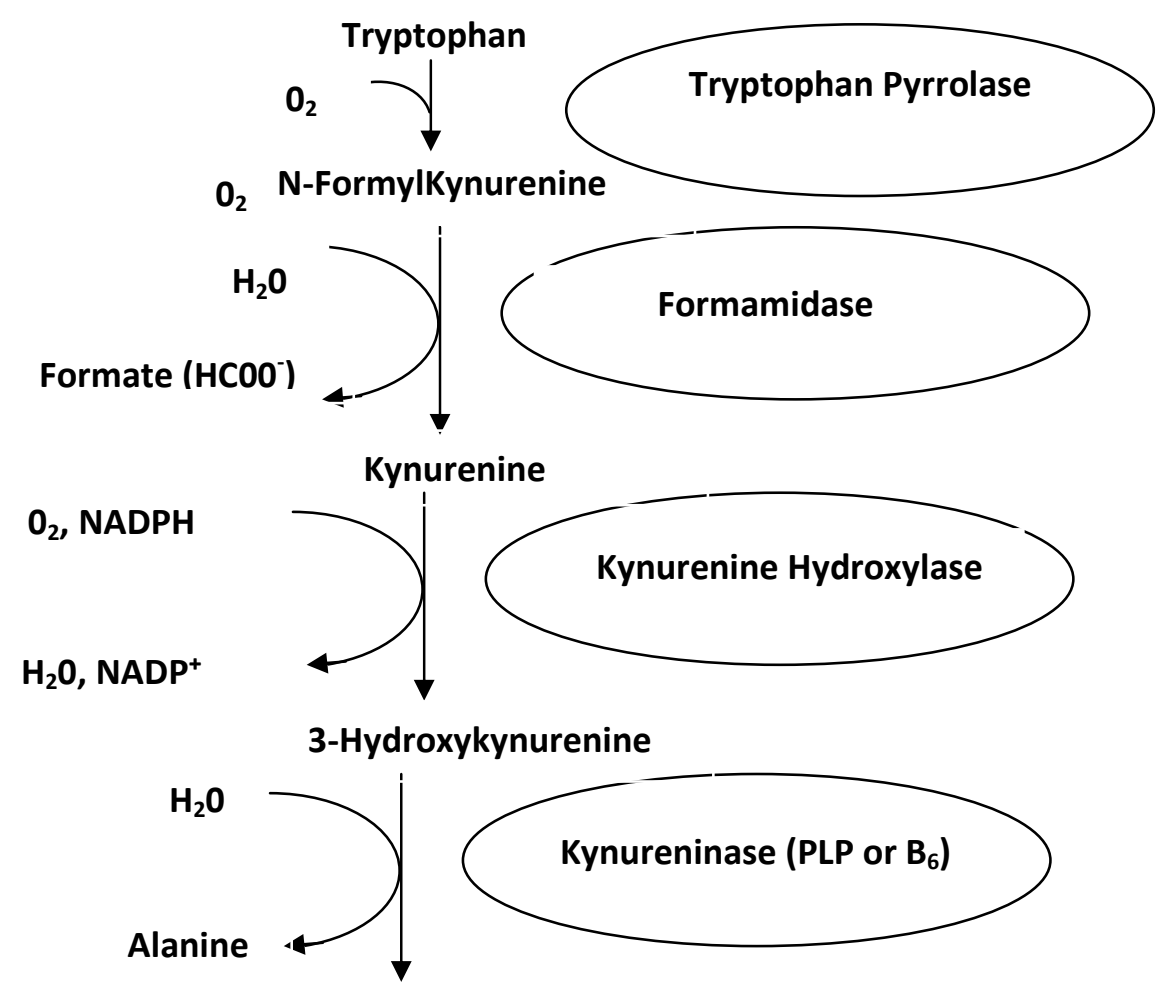

3-Hydroxyanthranilate

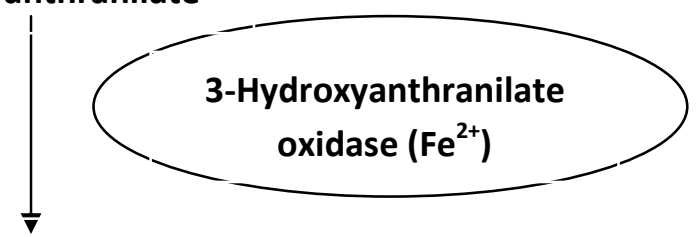

2-Amino-3-Carboxymuconate

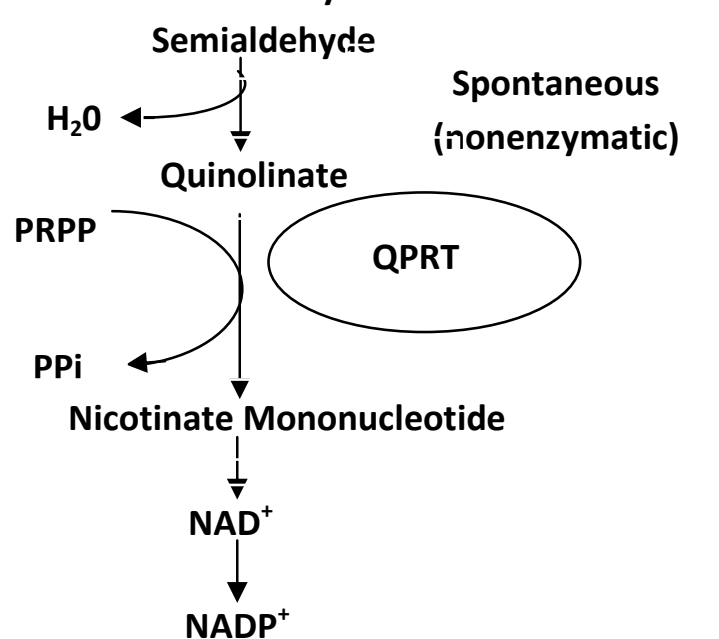

Fig. 1: Kynurenine pathway and its enzymes wifit are featured inside pear shape. PLP - Pyridoxal phosphate; QPRT - Quinolinate phosphoribosyl pyrophosphate transferase; PRPP Phosphoribosyl pyrophosphate.

Source: Satyanarayana and Chakrapani (2010) 
In Figure 1, Tryptophan pyrrolase oxygenates tryptophan to produce $\mathrm{N}$-formylkynurenine. Formamidase hydrolyses formylkynurenine to form kynurenine and formate as by-products. Kynurenine, in the presence of kynurenine hydroxylase, undergoes NADPH-dependent hydroxylation to give 3hydroxykynurenine. Kynurenunase, a pyridoxal phosphate (PLP)-dependent enzyme converts 3hydroxykynurenine into 3-hydroxyanthranilate and alanine as a by-product. 3-hydroxyanthranilate is cleared by $\mathrm{Fe}^{2+}$-dependent oxidase to form an unstable 2-amino-3-carboxymuconate semialdehyde, which in turn cyclizes spontaneously to form quinolinate. Quinolinate, in the presence of Quinolinate phosphoribosyltransferase is transformed into nicotinate mononucleotide. Nicotinate mononucleotide is a precursor for the biosynthesis of nicotinamide nucleotides, $\mathrm{NAD}^{+}$and $\mathrm{NADP}^{+}$, which are coenzymes of niacin.

\section{Serotonergic Pathway}

About $90 \%$ of the human body's total serotonin is produced in the enterochromaffin cells in the gut, where it is used to regulate intestinal movement (King, 2009) while the remainder is synthesized in serotonergic neurons of central nervous system (C.N.S) where it regulates mood, appetite, sleep, memory and learning (Berger et al, 2009). Serotonin secreted from the enterochromaffin cells eventually finds its way out of tissues into the blood for storage where it serves as a vasoconstrictor, helps to regulate homeostasis and blood clotting (Wikipedia, 2012b).

\section{Serotonergic Neurons}

These are nerve cells that produce serotonin in the C.N.S, brain, by an isoform of $\mathrm{TPH}_{2}$. The other one, $\mathrm{TPH}_{1}$, produces serotonin in the enterochromaffin cells or other tissues. Serotonergic neurons release serotonin into synapse that activates $5-\mathrm{HT}$ receptors located on the cell membrane of adjacent neuron. These receptors act as endogenous ligands that mediate the effects of serotonin and a broad range of pharmaceutical and hallucinogenic drugs. With the exception of the 5-hydroxytryptamine-3-receptor (5$\mathrm{HT}_{3}$ ), all other $5-\mathrm{HT}$ receptors activate an intracellular second messenger cascade. Serotonin action is terminated through the uptake of serotonin from the synapse. Most of the brain serotonin is not degraded after use, but collected by serotonin transporters on the surfaces of presynaptic serotonergic neurons. Such transporters include specific monoamine transporter (SERT), newly discovered monoamine transporter (PMAT) and others. If serotonergic neurons are abnormal in infants, there is a risk of sudden infant death syndrome (SIDS). Certain classes of drugs and pharmaceuticals that are used for the treatment of some human disorders target the $5-\mathrm{HT}$ system; such drugs include some antidepressants, antipsychotic, antiemetic, as well as the psychedelic drugs (Wikipedia, 2012b).

Figure 2 shows the various intermediates and enzymes which make up serotonin pathway.

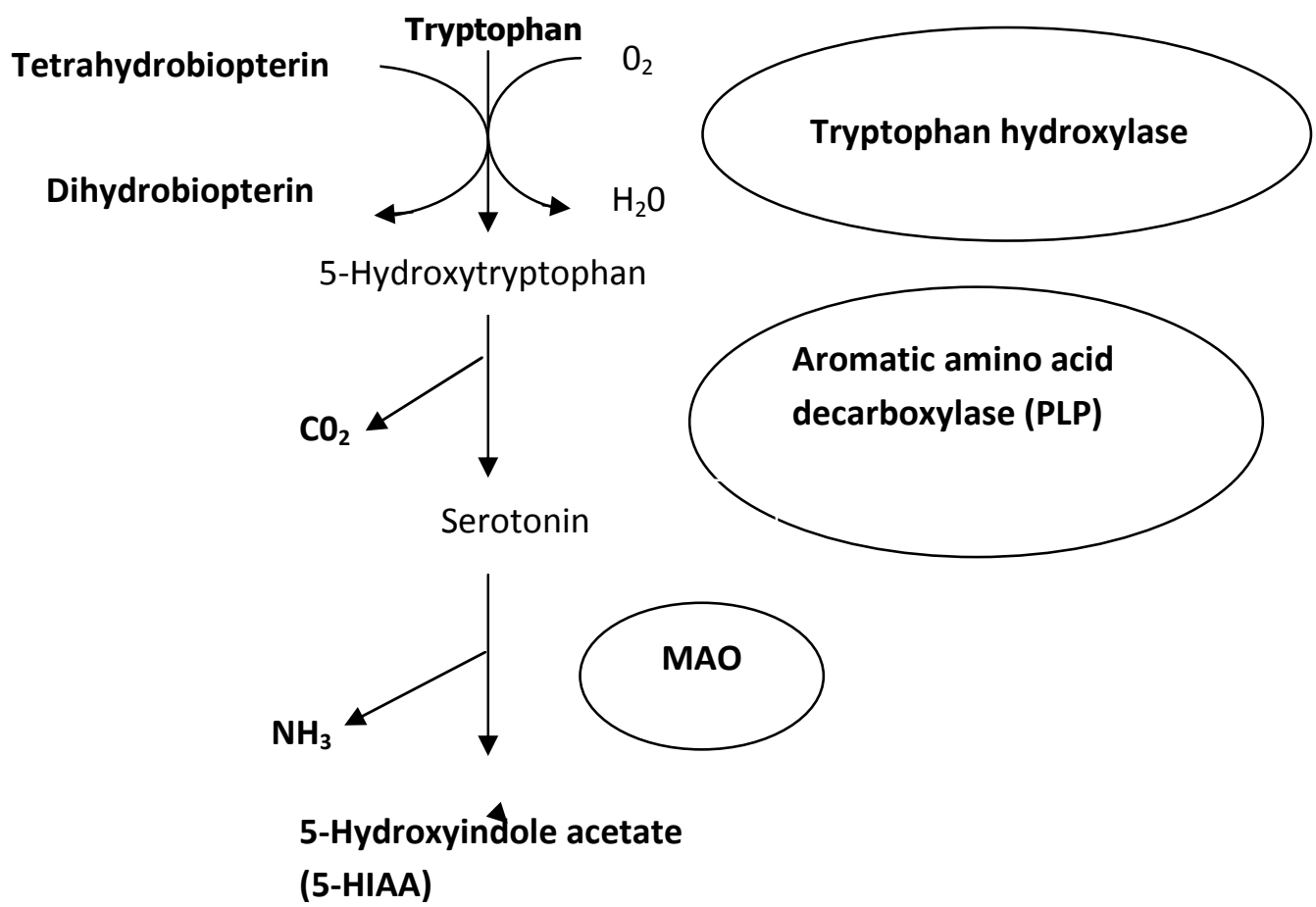

Fig. 2: Serotonin pathway and its enzymes which are featured inside pear shape.

PLP - Pyridoxal phosphate; MAO - Monoamine oxidase

Source: Satyanarayana and Chakrapani (2010) 
In Figure 2, tryptophan is hydroxylated by tryptophan hydroxylase, with tetrahydrobiopterin as a coenzyme, to form 5-hydroxytryptophan. 5-hydroxytryptophan is decarboxylated by pyridoxal phosphate-dependent aromatic amino acid decaboxylase to give serotonin. Monoamine oxidase (MAO) degrades serotonin to 5hydroindoleacetate (5HIA), which is excreted in urine.

\section{The Relevance of Kynurenine Pathway}

Kynurenine pathway serves several purposes such as: hepatic clearance mechanism for excess tryptophan, biosynthesis of nicotinamide $\left(B_{3}\right)$, cell-mediated immune system regulation, biosynthesis of quinolinate for hippocampus regulation (Pattern, 2012). Furthermore, the pathway helps to elucidate the prominent role of pyridoxal phosphate in tryptophan metabolism: kynureninase is a pyridoxal phosphate-dependent enzyme in the pathway, due to lack of this cofactor, the enzyme reaction is blocked and consequently 3hydroxykynurenine is diverted to form xanthurenate. Elevated excretion of xanthurenate serves as an indication of $B_{6}$ vitamin deficiency. The resulting defect in the activity of kynureninase causes reduced synthesis of $\mathrm{NAD}^{+}$and $\mathrm{NADP}^{+}$from tryptophan and with accompanied symptoms of pellagra (satyanarayana and Chakrapani, 2010).

\section{Relevance of Serotonin Pathway}

Serotonin pathway serves as a target for several classes of drugs such as antidepressants, antiemetic as well as psychedelic drugs to exert their effects. The pathway of serotonin synthesis is activated by tetrahydrobiopterin, $\mathrm{O}_{2}$, PLP, while inactivation can be caused by dihydrobiopterin. Serotonin, a bioactive product of SP, performs a variety of functions such as: a neurotransmitter across the synapse, a vasoconstrictor that helps in blood clotting, a stimulator of muscular contraction in the intestinal wall, a function that may be used for the development of antidote, a controller of mood, appetite, sleep, blood pressure, cognitive function e.t.c in the central nervous system. Serotonin increases mating behavior in male animals, an effect that may be harnessed to treat such sexual disorder as erectile dysfunction, hypo-sexuality in male human (Wikipedia, 2012b). It also serves for clinical diagnosis of patients with malignant carcinoid (Satyanarayana and Chakrapani, 2010). In addition, modulation of serotonin at synapses is thought to be a major action of several classes of pharmacological antidepressants (Wikipedia, 2012b).

\section{REFERENCES}

Berger M, Gray J.A and Roth B.L (2009). The Expanded Biology of Serotonin: Annual Review of Medicine. 60:355-66.

King M.W (2009). Serotonin. The Medical Biochemistry page. Indiana University School of Medicine. Retrived $1^{\text {st }}$ December, 2009 from http://.themedicalbiochemistry page.org/nerves.html\#5ht.

Pattern (2012). Organix profile pattern analysis. Retrieved $9^{\text {th }} \quad$ April, 2012 from http:// www.diagnosticinsight.com.au/medici/pattern

Satyanarayana U. and Chakprani U (2010). Meatabolism of amino acids. Biochemistry, $2^{\text {nd }}$ ed., Books and Allied (P) Itd., KOLKATA 700010, India. Pg 369374.

\section{Regulation of Kynurenine Pathway}

It is imperative for all metabolic activities in living organisms to be regulated to ensure their survival and well-being. As a result, kynurenine pathway is regulated by some of the following ways; the enzyme tryptophan pyrrolase is inducible. It is activated by tryptophan, glucocoticoids and glucagon hormones, $\mathrm{Fe}^{2+}$ and inactivated allosterically by NADH and NADPH. Kynureninase is activated by $A D P, P i$, pyridoxal phosphate, $\mathrm{Fe}^{2+}$ and inactivated by ATP. The formation of quinolinate is non-enzymatic and may be regulated by diverting 2-amino-3-carboxymuconate semialdehyde intermediate to an oxidative degradation by an enzymatic route such as picolinate carboxylase to form picolinic acid, which is common in cat (Satyanarayana and Chakprani, 2010).

\section{Dietary Source of Serotonin}

In human, serotonin levels are affected by diet. Foods that give an increased ratio of tryptophan to phenylalanine and leucine will increase serotonin levels. Food with a good ratio include the nuts of the walnut $(25-400 \mathrm{mg} / \mathrm{kg})$, plantain $(3-30 \mathrm{mg} / \mathrm{kg})$, bananas (3$30 \mathrm{mg} / \mathrm{kg})$, pineapple $(3-30 \mathrm{mg} / \mathrm{kg})$, tomatoes (3$30 \mathrm{mg} / \mathrm{kg}$ ) and others. Foods with a lower ratio such as whole wheat and rye bread inhibit the production of serotonin. Research also suggests consuming a diet rich in carbohydrates and low in protein will increase serotonin levels. However serotonin taken orally does not pass into the serotonergic pathways of the C.N.S system due to the blood-brain barrier, but its precursors, tryptophan and 5-hydroxytryptophan can and do cross the blood-brain barrier. These agents are available as dietary supplements, and may be effective serotonergic agents (Wikipedia, 2012b).

Conclusively, the study of the pathways gives insight to the understanding of abnormalities underlying pellagra. It also explains the diverse roles of tryptophan in the body in addition to protein synthesis such as the synthesis of nicotinamide nucleotides $\left(\mathrm{NAD}^{+}\right.$and $\mathrm{NADP}^{+}$), coenzymes of niacin, and serotonin. The study also reveals the glucogenecity of tryptophan by splitting off alanine, a precursor of glucose in gluconeogenesis and of prominence is of harnessing certain effects of serotonin for the treatment of intestinal poison and certain sexual dysfunctions in male human.

Stone T.W (2001). Kynurenines in the Central Nervous System: from endogenous obscurity to the rapeutic importance. Progress inNeurobiology. 64 (2): $185-218$

Walther D.J., Peter, J.U., Bashammakh, S., Hortnagl, H., Voits, M., Fink H. and Bader, M. (2003). Synthesis of serotonin by a second tryptophan hydroxylase isoform. Science 299 (5603): 76.

Wikipedia (2012a). Kynurenine pathway. Retrieved $9^{\text {th }}$ April, 2012 from http://en.wikipedia.org/wiki/kynureninepathway.

Wikipedia (2012b). Serotonin. Retrieved $9^{\text {th }}$ April, 2012 from http://.en.wikipedia.org/wiki/serotonin

Young S.N (2007). How to increase serotonin in the human brain without drugs. Review of Psychiatric Neuroscience. 32 (6): 394-99. 